\title{
A Matrix Adaptation Evolution Strategy for Optimization on General Quadratic Manifolds
}

\author{
Patrick Spettel and Hans-Georg Beyer \\ Supplementary Material
}

\section{FURTHER EXPERIMENTAL EVALUATION RESULTS}

For the quantitative evaluation, problems are generated by randomly sampling $N$ eigenvalues $\lambda_{j} \sim \mathcal{N}(0,1)$ for $j \in\{1, \ldots, N\}$ resulting in a quadratic constraint. Using those eigenvalues, a randomized $\mathrm{S}$ matrix is computed with $\mathbf{S}=\mathbf{U}^{T} \mathbf{D U}$, where $\mathbf{U}$ is also randomly generated such that $\mathbf{U}^{T} \mathbf{U}=\mathbf{I}$ and $\mathbf{D}=\operatorname{diag}\left(\lambda_{1}, \ldots, \lambda_{N}\right)$. To ensure that there are not only hyperbolically constrained problems, additional problems are considered: Absolute eigenvalues are considered to have additional elliptically constrained problems. In addition, half of the absolute eigenvalues are set to 0 to generate additional parabolic problems. For the objective function, a randomized ellipsoid function

$$
f(\mathbf{x})=\sum_{i=1}^{N} 10^{\frac{i-1}{N-1}}(\mathbf{R x}+\mathbf{t})_{i}^{2}
$$

is used, where $\mathbf{R}_{j k} \sim \mathcal{N}(0,1)$ and $\mathbf{t}_{j} \sim \mathcal{N}(0,1)$. ECDF plots are shown in Figure 5 for dimension 10, Figure 6 for dimension 20 , Figure 7 for dimension 30, and Figure 8 for dimension 40. The optimal values used as a reference for creating the ECDF plots are determined by solving the randomly generated optimization problems using fmincon. 

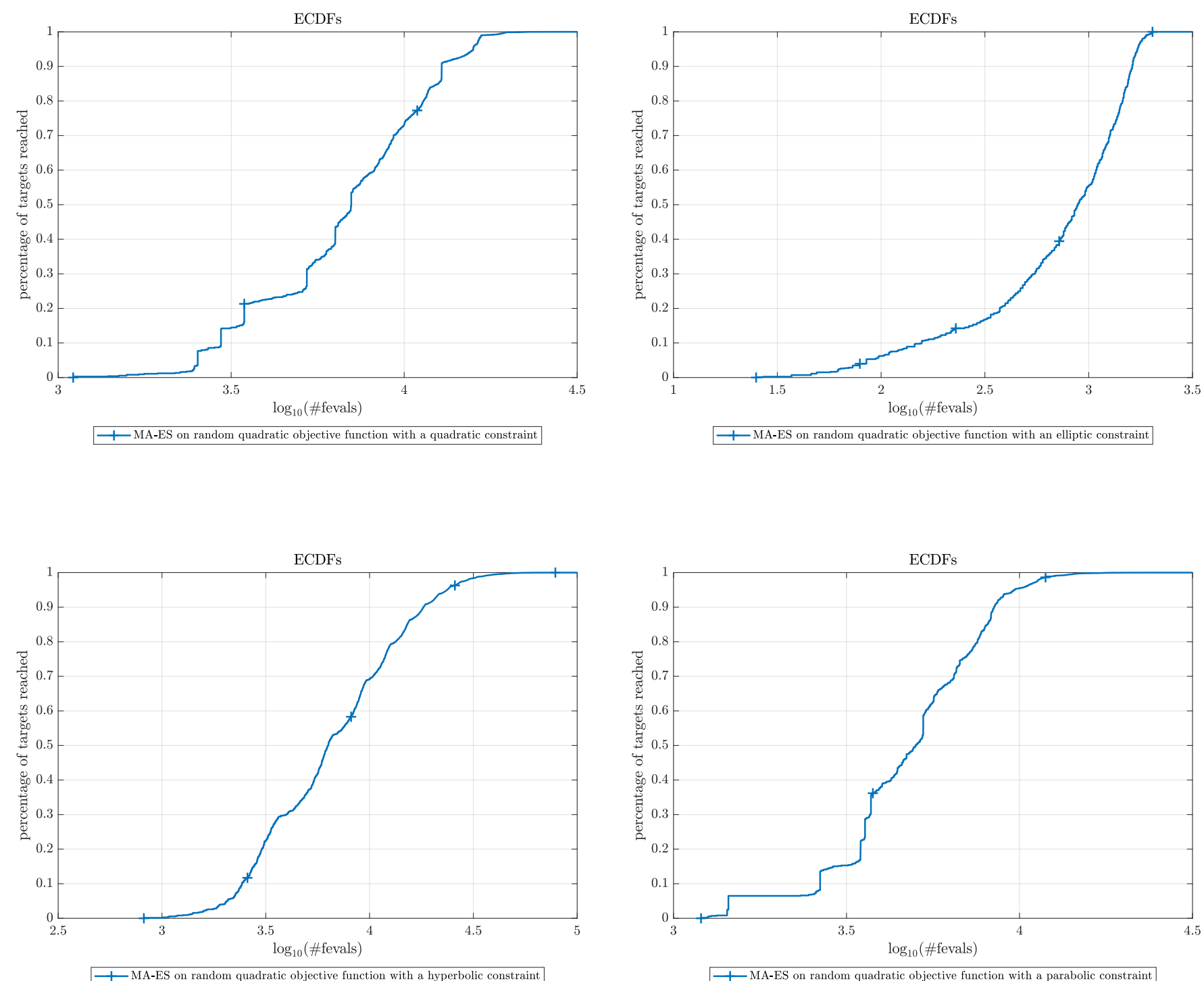

Figure 5: Results (as ECDF plots) of applying the proposed algorithm to the randomly generated problems for dimension 10. 

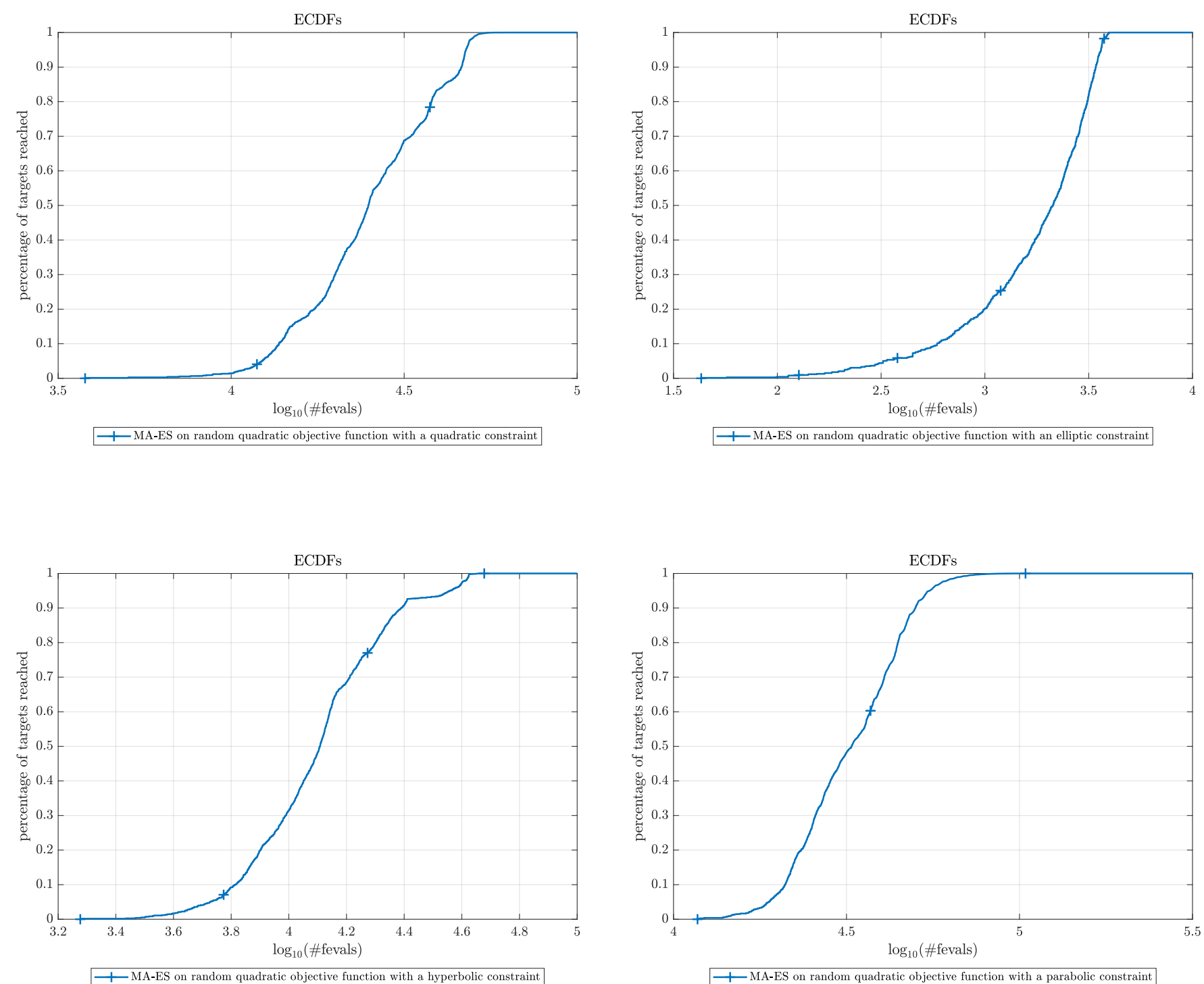

Figure 6: Results (as ECDF plots) of applying the proposed algorithm to the randomly generated problems for dimension 20. 

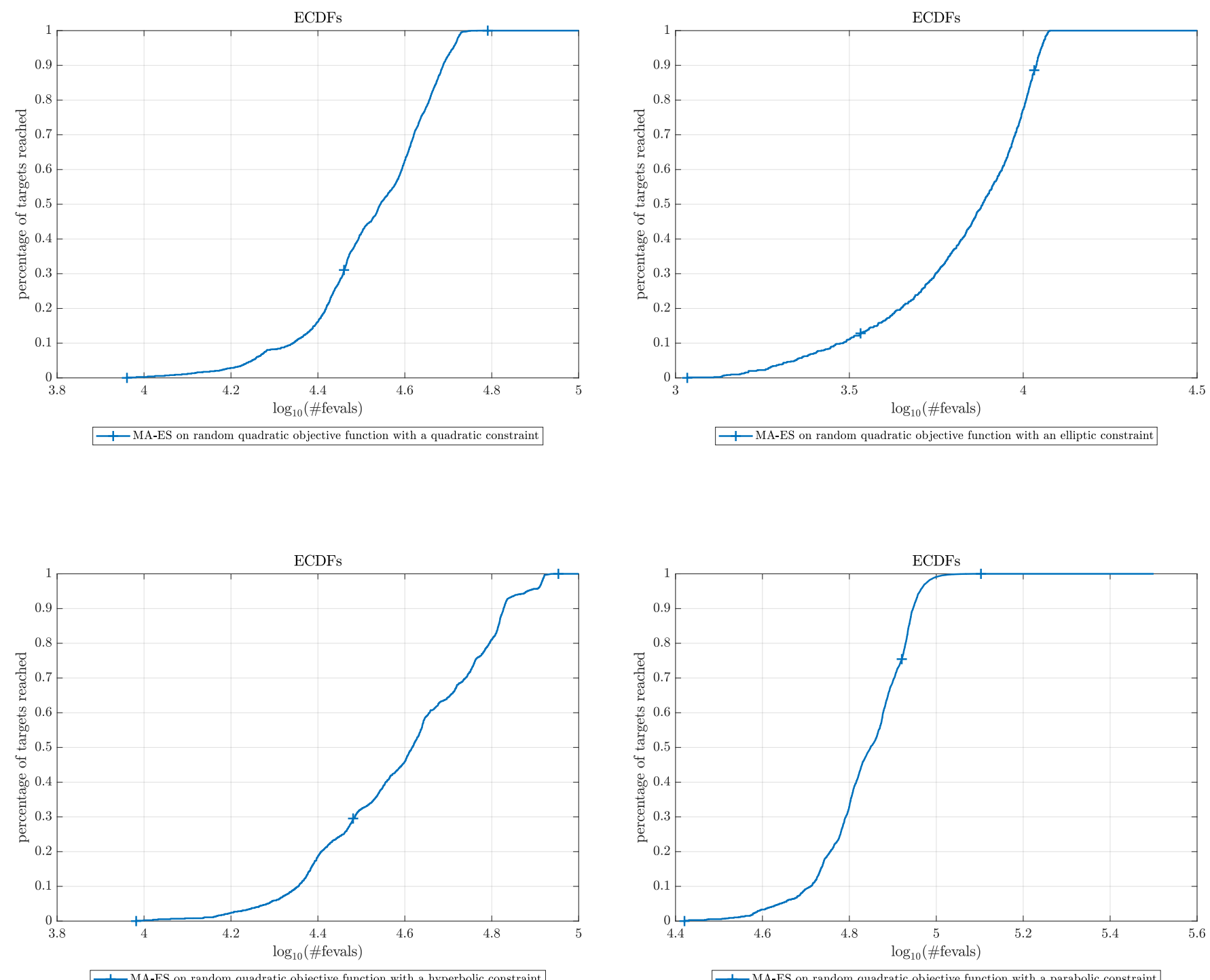

Figure 7: Results (as ECDF plots) of applying the proposed algorithm to the randomly generated problems for dimension 30. 

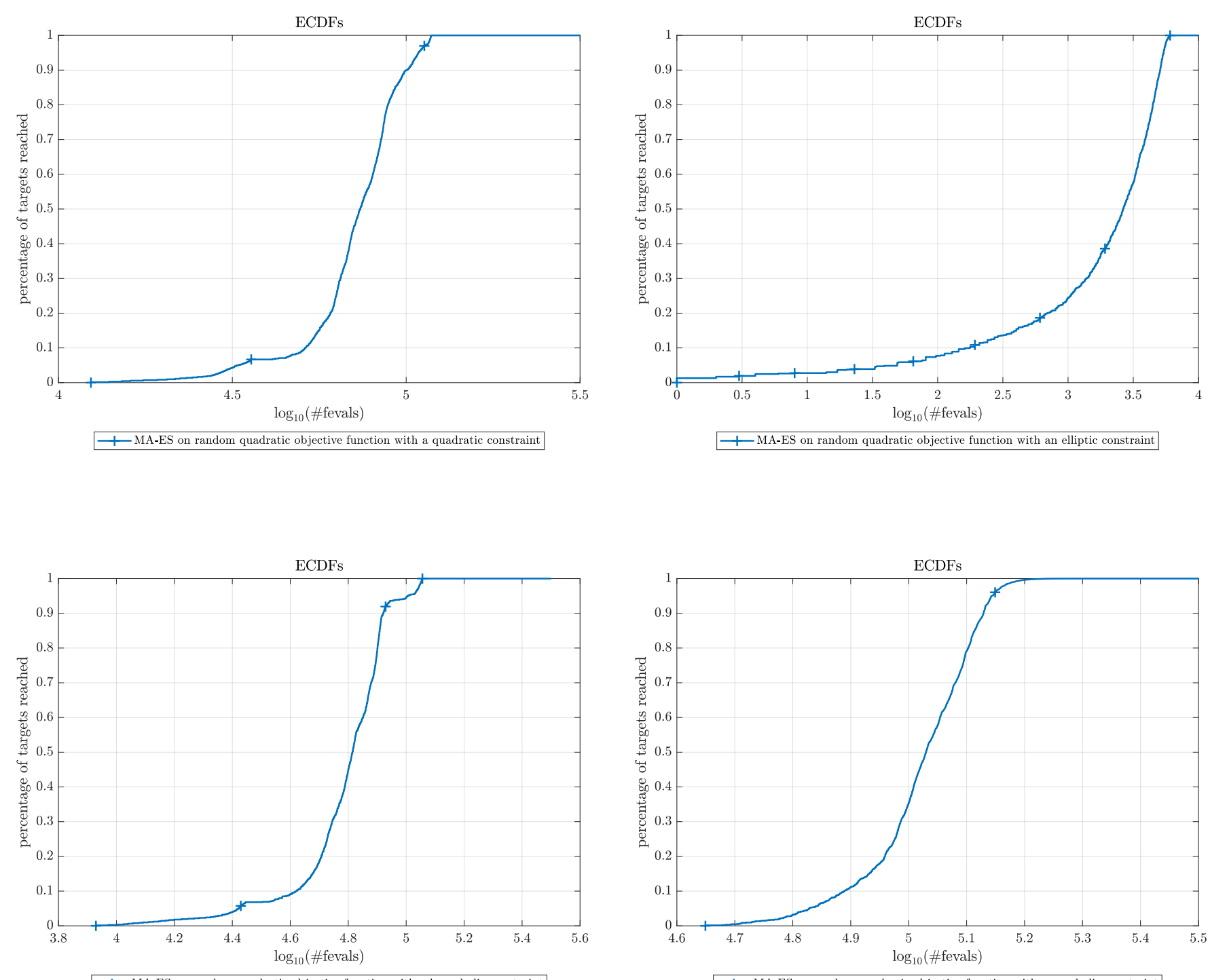

Figure 8: Results (as ECDF plots) of applying the proposed algorithm to the randomly generated problems for dimension 40. 